\title{
Distribution of cervical intraepithelial neoplasia: are hysterectomy specimens sampled appropriately?
}

\author{
M Heatley
}

\begin{abstract}
Aim-To determine whether sampling the anterior and posterior cervical lips in hysterectomy specimens provides the best opportunity for detecting unsuspected cervical intraepithelial neoplasia (CIN). Methods-The frequency with which CIN was identified at a range of positions on the anterior and posterior lips and left and right lateral aspects of the cervix was assessed in 100 cone biopsy specimens.

Results-CIN affected one or other cervical lip in all 100 cases studied and involved the midline positions (12 and 6 o'clock) in 94. The lateral edges of the cervical canal were also involved in 38 cases.

Conclusion-CIN is more likely to be identified on the anterior and posterior lips than on the lateral aspect of the cervical os. The findings support the continuation of the established practice of taking blocks from the midline in these areas. (f Clin Pathol 1995;48:323-324)
\end{abstract}

Keywords: Cervix, biopsy, tissue processing.

Protocols describing the gross dissection of a hysterectomy specimen removed for a nonmalignant condition usually recommend that, as a minimum, two midline blocks of cervix, one from the anterior and one from the posterior lip, should be examined histologically. ${ }^{1-3}$ Cervical intraepithelial neoplasia (CIN) is not macroscopically detectable ${ }^{4}$ and the purpose of examining these random histological sections \section{Accepted for publication 25 August 1994 \\ Department of Pathology, University PO Box 596, Beech Hill Road, Sheffield S10 2UL Dr M Heatley}

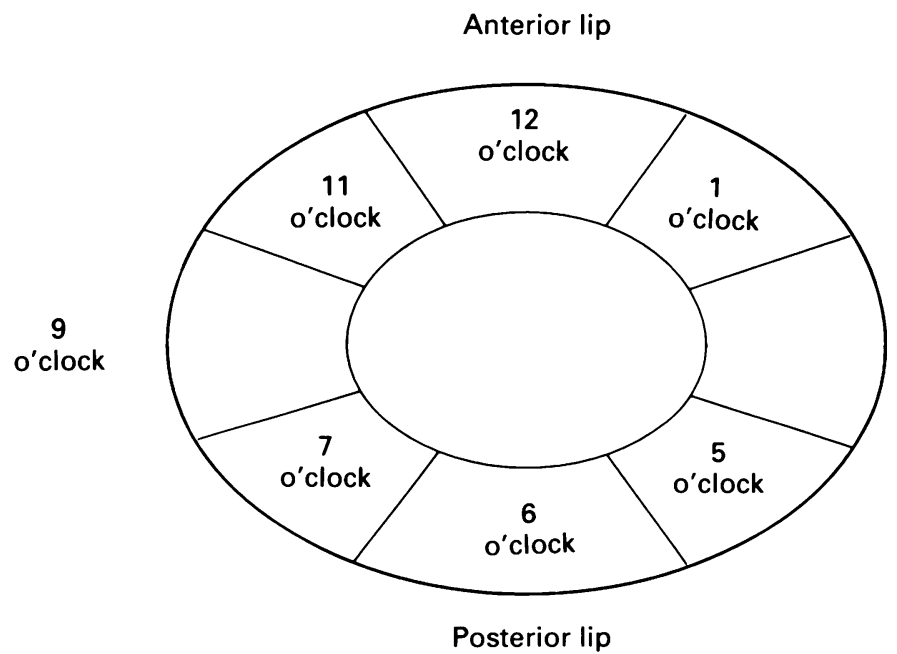

3

Positions at which dysplasia was identified as occurring in 100 cone biopsy specimens of cervix dissected according to the method of Foote and Stewart. ${ }^{5}$ is to detect previously unsuspected CIN. This contrasts with the more systematic approach of processing the entire cervix when CIN has been proven to be present on either a preoperative punch biopsy or a cervical smear.

The validity of assuming that examination of midline blocks of the anterior and posterior lips of cervix provides the best chance of detecting previously unsuspected cervical intraepithelial neoplasia was assessed in this study.

\section{Methods}

One hundred cone biopsy specimens of cervix were examined, including diathermy loop and laser excisions and cold knife biopsy specimens. Before dissection, a colour print of the macroscopic specimen was made using a Sony UP2200 video printer system. ${ }^{4}$ The specimens were serially sectioned beginning from one lateral edge using the method of Foote and Stewart. $^{5}$ The assumed relative positions of the cervical lips and the position from which each portion of tissue originated was carefully recorded on the print. Each block of tissue was placed in a processing cassette which was labelled with a unique identifying letter. This method, rather than the alternative of radially sectioning the cervix, was chosen since it avoids the problems of interpretation which may arise when abnormal epithelium lies on the narrow margin of a wedge shaped block. It also facilitates assessment of tumour volume in a malignant lesion if this is small.

The blocks were processed on a Miles Scientific Tissue Tek vacuum impregnation processor 1000 using a standard processing schedule and embedded in high quality paraffin wax. Haematoxylin and eosin stained sections cut at $5 \mu \mathrm{m}$ intervals on a microtome were examined by a single pathologist. The presence and grade ${ }^{6}$ of squamous CIN in each of the histological secions, and its position, were recorded on the macroscopic image.

The site of CIN in each specimen was mapped according to a predetermined plan (figure). Positions were identified cor-

Table 1 The involvement of the various anatomical sites of the cervix in 100 cone biopsy specimens with cervical intraepithelial neoplasia

\begin{tabular}{lc}
\hline Site & Cases \\
\hline One cervical lip & 18 \\
Both lips & 40 \\
One lips and one lateral edge & 6 \\
One lip and both lateral edges & 1 \\
Both lips and one lateral edge & 23 \\
Both lips and both lateral edges & 8 \\
Circumoral distribution of dysplasia & 4 \\
\hline
\end{tabular}


Table 2 The distribution of cervical intraepithelial neoplasia, by histological grade, in 100 consecutive cone biopsy specimens of cervix

\begin{tabular}{|c|c|c|c|c|c|c|c|c|}
\hline \multirow[b]{2}{*}{ Grade of dysplasia } & \multicolumn{3}{|l|}{ Anterior lip } & \multicolumn{3}{|c|}{ Posterior lip } & \multicolumn{2}{|l|}{ Lateral edges } \\
\hline & 11 o'clock & 12 o'clock & 1 o'clock & 5 o'clock & 6 o'clock & 7 o'clock & First (3 o'clock) & Second (9 o'clock) \\
\hline $\begin{array}{l}\text { Negative } \\
\text { Basal nuclear } \\
\text { abnormality of } \\
\text { undetermined }\end{array}$ & 22 & 19 & 34 & 39 & 29 & 32 & 72 & 66 \\
\hline $\begin{array}{l}\text { significance } \\
\text { CIN } 1 \\
\text { CIN } 11 \\
\text { CIN } 111\end{array}$ & $\begin{array}{r}5 \\
27 \\
21 \\
23\end{array}$ & $\begin{array}{r}8 \\
23 \\
17 \\
32\end{array}$ & $\begin{array}{r}9 \\
23 \\
13 \\
21\end{array}$ & $\begin{array}{r}7 \\
20 \\
17 \\
17\end{array}$ & $\begin{array}{r}8 \\
18 \\
17 \\
26\end{array}$ & $\begin{array}{r}7 \\
16 \\
20 \\
23\end{array}$ & $\begin{array}{l}5 \\
8 \\
6 \\
8\end{array}$ & $\begin{array}{r}2 \\
15 \\
6 \\
9\end{array}$ \\
\hline Total & 98 & 99 & 100 & 100 & 98 & 98 & 99 & 98 \\
\hline
\end{tabular}

responding to the 11,12 , and 1 o'clock positions on the anterior lip, the 5,6 , and 7 o'clock positions on the posterior lip, and the 3 o'clock and 9 o'clock positions representing the left and right lateral positions, respectively. The frequency with which CIN was identified at each of these sites was then calculated.

\section{Results}

CIN affected one or other lip of the cervix in all 100 cases studied and in 38 cases it also involved one or other lateral edge of the parous cervical os. In an additional four cases the entire circumference of the cervical os was involved (table 1). The frequency with which CIN was identified at each of the eight sites is recorded in table 2 . It was present at either the 12 o'clock or the 6 o'clock position, the usual site from which blocks are taken in hysterectomy specimens, in $94 \%$ of cases. Cervical intraepithelial neoplasia was identified with greater frequency at these sites and tended to be of a higher grade than that on the lateral aspects of the cervical os (the 3 o'clock and 9 o'clock positions).

\section{Discussion}

The purpose of this study was to confirm that the routine practice of sampling the anterior and posterior lips of the uterine cervix in the midline was an efficient screening test and provided the maximum probability of sampling previously unsuspected CIN. The hypothesis was tested by studying the site at which CIN was present in 100 consecutive cone biopsies of cervix performed as part of the treatment of CIN. The study showed that dysplasia occurred in one or other of these two blocks in $94 \%$ of the cases studied, reflecting the findings in previous colposcopic ${ }^{7}$ and histological ${ }^{489}$ studies.

While the current sample probably originates from a different, younger population than those having a hysterectomy for uterine prolapse or dysfunctional uterine bleeding, the alternative of submitting serial sections of the entire cervix from "routine" hysterectomy specimens would have been impracticable. Unsuspected CIN in such specimens is rare as, in this unit, patients undergoing hysterectomy for benign disease have a preoperative smear test to exclude dyskariosis or malignancy. Consequently it would have been necessary to examine a large number of cases before encountering an adequate number of cases of undiagnosed cervical intraepithelial neoplasia after hysterectomy. By selecting a group with a high probability of having a cervical intraepithelial lesion we maximised our ability to study the distribution of such lesions.

The second alternative was to examine the cervix from Wertheim hysterectomy specimens. These are also routinely submitted in total but usually after a cold knife or diathermy loop excision of the transformation zone has removed an area of in situ or invasive malignancy and distorted the cervical architecture.

The technique of specimen dissection used in this study ${ }^{4}$ is a common technique for assessing the extent of dysplasia in cervical cone biopsy specimens. It does not permit a direct comparison with the alternative method of ascribing dysplasia to a site on the cervix corresponding to the position on the face of a clock. ${ }^{2}$ For example it was not possible to identify epithelium at the $2,4,8$, or 10 o'clock positions in the present study. This alternative method of cutting the cervix radially ${ }^{2}$ was not used as it may be followed by distortion of the transformation zone with separation of the epithelium from the underlying stroma, and in the epithelium being underrepresented in some blocks.

The findings in this study confirm that dysplasia is more likely to be identified on the anterior and posterior lips than on the lateral aspects of the cervical os. This is in keeping with the colposcopic and clinicopathological correlative findings of other investigators and supports the convention of routinely sampling these sites.

1 Rosai J. Guidelines for handling most common and important surgical specimens. In: Rosai J, ed. Ackerman's surgical pathology, 7th ed. St Louis: CV Mosby, 1989:1946.

2 Robboy SJ, Kraus FT, Kurman RJ. Gross description, processing and reporting of gynaecologic and obstetric specimens. In: Kurman RJ, ed. Blaustein's pathology of the female genital tract, 3rd ed. Berlin: Springer Verlag, 1987:925-40.

3 Scurry J, Patel K, Wells M. Gross examination of uterine specimens. F Clin Pathol 1993;46:388-93.

4 Russel L, Appleyard J, Heatley MK. The application of the Sony UP-2200 colour video printer system to specimen photography [abstract]. $\mathcal{F}$ Pathol 1994;172(suppl):129A

5 Foote FW, Stewart FW. The anatomical distribution of intraepithelial epidermoid carcinomas of the cervix. Cancer 1948;1:431-9.

6 Anderson MC, Brown GL, Buckley CH, Fox H, Jenkins D Lowe DG, et al. Current views of cervical intraepithelia neoplasia. $f$ Clin Pathol 1991;44:969-78.

7 Richart RM. Colpomicroscopic studies of the distribution of dysplasia and carcinoma in situ on the exposed portion of the uterine cervix. Cancer 1965;18:950-4.

$8 \mathrm{Ng}$ ABP, Reagan JW. Microinvasive carcinoma of the uterine cervix. Am f Clin Pathol 1969;52:511-29.

9 Przybora LA, Plutowa A. Histological topography of carcinoma in situ of the cervix uteri. Cancer 1959;12:263-77.
comoly 